\title{
ALTERAÇÃO ESTRUTURAL DE UMA ÁREA FLORESTAL EXPLORADA CONVENCIONALMENTE NA BACIA DO PARAÍBA DO SUL, MINAS GERAIS, NOS DOMÍNIOS DE FLORESTA ATLÂNTICA ${ }^{1}$
}

\begin{abstract}
Ana Patrícia Cota Gomes² ${ }^{2}$ Agostinho Lopes de Souza ${ }^{3}$, João Augusto Alves Meira Neto ${ }^{4}$
RESUMO - O presente estudo, realizado no município de Matias Barbosa,MG, na Fazenda Paciência, teve como objetivo avaliar as alterações estruturais ocorridas em uma Floresta Estacional Semidecidual, nos domínios de floresta Atlântica, em decorrência das atividades de exploração convencional de um plano de manejo. Para isso, foi utilizado um trecho contínuo de floresta que teve uma área explorada há seis anos e outra área não-explorada. Foram lançadas parcelas para estudo da vegetação arbórea, nas quais se avaliou a sua estrutura. Os dados foram analisados de forma comparativa, considerando-se a situação observada na área de floresta não-explorada como desejável. O resultado do estudo indicou que a atividade de exploração madeireira realizada na Fazenda Paciência tem levado a alterações significativas na estrutura, além de um depauperamento do valor comercial do estoque remanescente da área de floresta explorada, podendo levar a um comprometimento dos recursos da comunidade florestal.
\end{abstract}

Palavras-chave: Indicadores, estrutura fitossociológica, Floresta Estacional Semidecidual, plano de manejo, exploração florestal.

\section{STRUCTURAL CHANGES IN A CONVENTIONALLY EXPLOITED FOREST IN THE PARAÍBA DO SUL BASIN, STATE OF MINAS GERAIS, BRAZIL, IN THE ATLANTIC FOREST DOMAIN}

\begin{abstract}
The objectives of this study were to evaluate the structural changes caused by the activities of a conventional management plan in a semideciduous forest in the Atlantic Forest domain. The study was conducted in the Fazenda Paciência, municipality of Matias Barbosa, Minas Gerais state, Brazil. A continuous stretch of forest was analyzed, which contained areas that were exploited six years ago and areas that remained untouched. Plots were established to survey the floristic and structural composition of the arboreal vegetation. Data were analized in a comparative way and the state of the non-exploited forest was considered as the desirable one. The study results indicated that the harvesting as it was done at the Fazenda Paciência was causing significant changes in the floristic and structural composition, aside from decreasing the commercial value of the remaining stock, which could endanger the forest resources of the area.
\end{abstract}

Key words: Indicators, Phytosociologic structure, semideciduous seasonal forest, forest exploitation, forest management.

\footnotetext{
${ }^{1}$ Recebido para publicação em 19.3.2003 e aceito para publicação em 08.6.2004.

${ }^{2}$ Engenheira Florestal, M. S. em Ciência Florestal pela Universidade Federal de Viçosa e coordenadora do Programa de Treinamento e Capacitação do Imaflora. Cx. Postal 411, Cep: 13400-970 Piracicaba, SP. E-mail: <patricia@imaflora.org / patriciacotagomes@yahoo.com.br>.

${ }^{3}$ Prof. Titular de Manejo de Florestas Nativas, no Departamento de Engenharia Florestal da Universidade Federal de Viçosa Cep: 36570-000. E-mail: <alsouza@ufv.br>.

${ }^{4}$ Prof. Adjunto II de Sistemática das Espermatófitas, no Departamento de Biologia Vegetal da Universidade Federal de Viçosa Cep: 36570-000.E-mail: <j.meira@ufv.br>.
} 


\section{INTRODUÇÃO}

O homem, historicamente, sempre exerceu uma grande pressão diante dos recursos naturais. Hosokawa et al. (1998) citaram o historiador Perlin (1989) em sua obra $A$ forest journey, em que relatou que "à medida que uma civilização se desenvolvia, as florestas desapareciam e que as mesmas se regeneravam, quando a dita civilização entrava em decadência”.

Nos últimos anos, o desejo de que o uso dessas florestas se faça de forma "ecologicamente adequada, economicamente sustentável e socialmente justa” tem sensibilizado inúmeras organizações e mobilizado uma multidão, com o objetivo de colocar em prática o paradigma do "bom manejo" (RODRIGUEZ, 1998). Em razão disso, tem-se buscado um modelo de desenvolvimento florestal que visa substituir o antigo modelo de exploração, com grandes extrações volumétricas, às vezes a partir de corte raso e alteração de uso do solo, por um modelo moderno de manejo florestal de rendimento sustentável, no qual as interações das florestas com outros recursos, como água, solo, atmosfera, fauna e conservação da biodiversidade e capacidade de renovação, são levados em consideração (COELHO, 1999).

Muitos dos Planos de Manejo Florestal Sustentável (PMFS) atualmente em andamento no Brasil apresentam, porém sérios problemas técnicos e de execução. Grande parte desses não passa de exploração florestal convencional rotulada de plano de manejo, ou seja, executada sem planejamento e utilização de técnicas de Exploração de Impacto Reduzido (EIR) e, sobretudo, sem efetiva aplicação de tratamentos silviculturais.

De acordo com o diagnóstico técnico dos planos de manejo realizados pela Embrapa (1996) para a região de Paragominas, no Estado do Pará, foi constatado que o manejo está sendo usado apenas para satisfazer as exigências legais, não ocorrendo manejo de fato, pois a execução é falha e não atende à legislação vigente nem ao programado nos projetos, concluindo-se, por fim, que a atividade madeireira na região é puramente extrativista.

O Ibama (1999), por sua vez, durante o processo de análise dos PMFS detectou uma série de motivos que levou à suspensão desses planos. Entre os problemas levantados, citam-se os de ordem técnica, como: falta de responsável técnico; não realização de atividades prescritas no projeto; falta de manutenção dos limites dos talhões e de sua identificação; não implantação de tratamentos silviculturais previstos nos PMFS; não observância do diâmetro mínimo de corte; exploração em área de reserva legal e, ou, em área de preservação permanente; exploração sem planejamento, com altos impactos à vegetação remanescente; e não cumprimento do previsto nos PMFS.

Problemas como a falta de capacitação de profissionais que atuam na elaboração, execução e fiscalização desses planos, bem como a falta de recursos financeiros destinados às vistorias e acompanhamentos dos planos de manejo, agravam a situação, colaborando com a idéia equivocada de que não é possível utilizar os recursos florestais com o mínimo de impacto, considerando-se os aspectos sociais, econômicos e ambientais.

Conhecer e quantificar os impactos na vegetação após as atividades de manejo constituem-se em uma importante ferramenta na avaliação da "performance” dos planos de manejo florestal. Essa avaliação permite a prescrição de medidas mitigadoras e ações que irão atuar, de forma pontual, reduzindo os custos e viabilizando a execução de um plano de manejo com um mínimo de impacto à organização e funcionamento da comunidade vegetal.

Dentro desse contexto, o objetivo deste estudo foi avaliar as alterações estruturais ocorridas em um trecho de Floresta Estacional Semidecidual, nos domínios de floresta Atlântica (VELOSO, 1992), em decorrência das atividades de exploração madeireira convencional de um plano de manejo.

\section{MATERIAL E MÉTODOS}

\section{1. Área de Estudo}

O presente trabalho foi realizado na Fazenda Paciência, situada entre os municípios de Matias Barbosa e Juiz de Fora, no Vale do Paraíba do Sul, Estado de Minas Gerais.

De acordo com o plano de zoneamento elaborado em 1957 para a Fazenda Paciência, a área de floresta estudada encontrava-se ocupada por agricultura e pastoreio (HERZOG,1957), sendo, portanto, um fragmento florestal secundário, regenerado após essas 
atividades.

Naquela propriedade vem sendo executado, desde 1994, um PMFS, objetivando a produção de madeira para serraria voltada, principalmente para a fabricação de carrocerias de caminhão. O referido PMFS vem sendo executado num trecho de 291 ha de floresta, com autorizações de corte seletivo de talhões anuais de 24,25 ha, com a intensidade de corte de 37,26 m³ ha de volume comercial (SOUZA et al.,1998).

Para a realização deste estudo foi utilizada uma área contínua de floresta de 100 ha, a qual teve duas condições de exploração: uma área explorada convencionalmente há seis anos e outra que permaneceu inexplorada. O estudo conduzido partiu do pressuposto de que se trata de duas áreas de uma mesma comunidade florestal, de mesma idade, florística e estruturalmente muito semelhante. No entanto, ambas não são idênticas, pelo fato de se tratar de áreas com diferentes históricos de perturbações antrópicas, representadas pelas intervenções de exploração madeireira.
Com as devidas considerações, procurou-se estabelecer comparações entre as áreas de florestas explorada e não-explorada, buscando estimativas de parâmetros de composição e estrutura que servissem como indicadores da natureza das alterações decorrentes das atividades de exploração florestal convencional.

\subsection{Amostragem}

Para a avaliação da vegetação arbórea, foram estabelecidas e medidas 10 parcelas de área fixa de $1.000 \mathrm{~m}^{2}$ (50 x $20 \mathrm{~m}$ ) cada, totalizando 1 ha de amostra, sendo 0,5 ha de amostra para a área de floresta explorada e 0,5 ha para a área de floresta não-explorada. A locação dessas unidades amostrais no campo foi feita com o auxílio de uma bússola, de forma sistemática, a cada $20 \mathrm{~m}$, dispostas paralelamente ao longo das curvas de nível do terreno, em que se procurou amostrar ambas as áreas de floresta, o mais homogeneamente possível, levando em consideração a topografia local, o que permitiu, assim, estudos comparativos entre as duas condições de floresta (Figura 1).

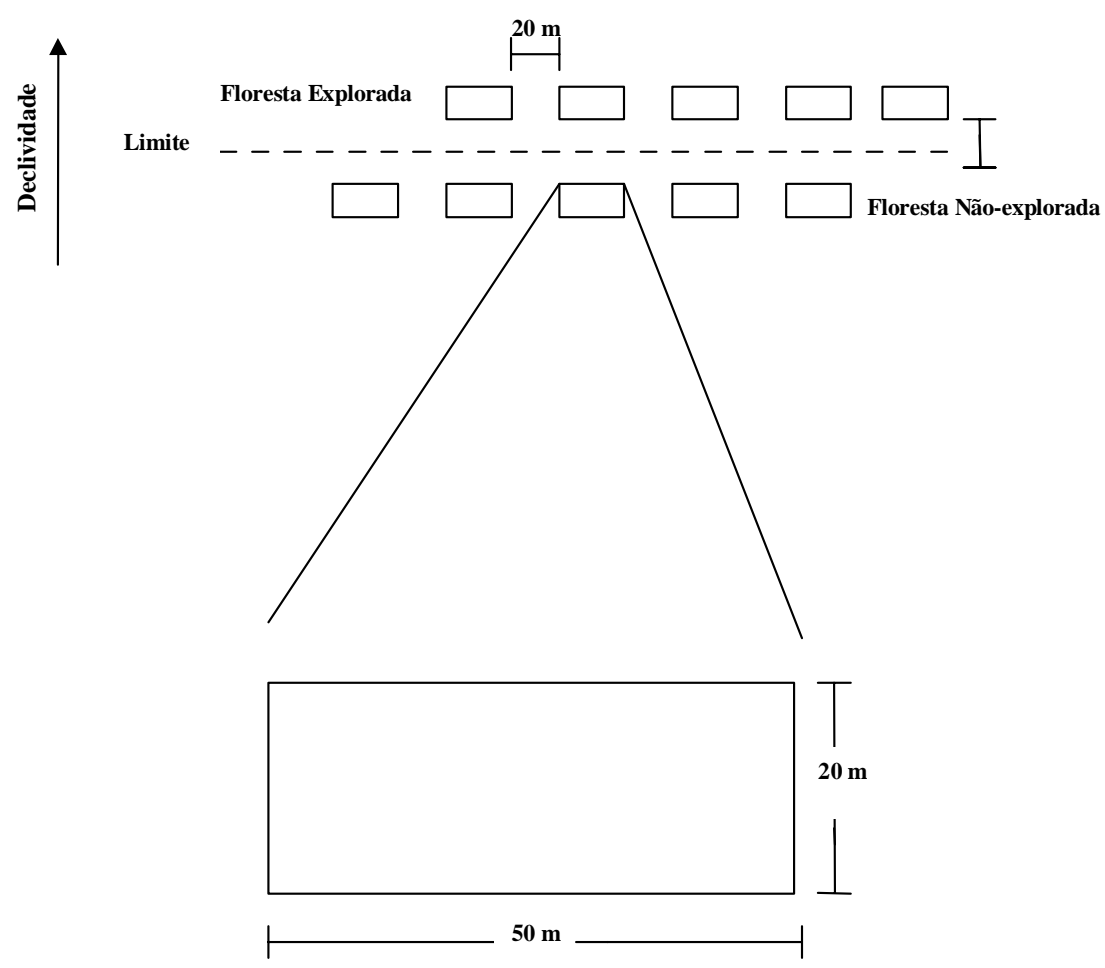

Figura 1 - Desenho esquemático das unidades amostrais na área estudada, Fazenda Paciência, no município de Matias Barbosa, Minas Gerais.

Figure 1 - Schematic drawing of the sampled units, designed for Fazenda Paciência, municipality of Matias Barbosa, Minas Gerais State. 


\subsection{Análise Estrutural}

Todos os indivíduos arbóreos encontrados nas parcelas, com DAP (diâmetro do tronco à altura de $1,30 \mathrm{~m}$ do solo) superior ou igual a $10 \mathrm{~cm}$, foram identificados, quando possível, por seu nome vulgar regional e tiveram seu material botânico coletado para identificação taxonômica, sendo posteriormente anexado ao Herbário VIC, da Universidade Federal de Viçosa, MG. Nesses indivíduos foram feitas medições do DAP, estimativa de altura e classificação das espécies por grupo de uso, sendo: espécies comerciais, espécies com potencial madeireiro e demais espécies.

Além disso, estudou-se a estrutura interna, conforme proposto por Silva e Lopes (1984), classificando as árvores amostradas com relação a:

a)Infestação de cipós: em nenhuma, somente no tronco, somente na copa e no tronco e na copa.

b) Posição de danos: nenhum, no tronco e na copa.

c) Qualidade de danos: nenhum, devido a causas naturais (fauna, flora, ventos e outros) e devido à exploração.

d) Infestação de bambus: nenhuma, no tronco e na copa.

Os parâmetros estimados foram os usuais em estudos fitossociológicos, descritos por MuellerDombois e Ellenberg (1974), como densidade absoluta $\left(D A_{i}\right)$ e dominância absoluta $\left(D o A_{i}\right)$, além do Volume de Fuste com Casca $\left(V o A_{f c c}\right)$. A partir desses dados foram realizadas ainda as análises das distribuições de $D A_{i j}, D o A_{i j}$ e $V o A_{f c c}$ por hectare, pora a i-éspécie na j-ésima classe de diâmetro, com o intervalo entre classes de $5 \mathrm{~cm}$.

\subsection{Análise Estatística}

Adotou-se o delineamento inteiramente casualizado, com seis tratamentos, resultantes da combinação de duas condições de exploração (área de floresta não-explorada e área de floresta explorada) e três grupos de uso (espécies comerciais, espécies com potencial madeireiro e outras espécies). Para as variáveis em estudo, foram aplicados os testes de normalidade de Lilliefors (LILIEFORS, 1967) e os de homogeneidade das variâncias de Cochran e Bartlett (WINER, 1962).

\section{RESULTADOS E DISCUSSÕES}

\subsection{Estrutura Horizontal}

\subsubsection{Densidade Absoluta $\left(D A_{i}\right)$}

A área da floresta não-explorada apresentou 786 indivíduos por hectare, em que as cinco espécies de maiores densidades, que totalizaram juntas 36,64\% do total de indivíduos, foram: Xylopia sericea (146 indivíduos/ha), Anadenanthera colubrina (52 indivíduos/ha), Machaerium nyctitans (50 indivíduos/ha), Apuleia leiocarpa e Platypodium elegans (40 indivíduos/ha).

Na área da floresta explorada, a densidade total foi de 696 indivíduos por hectare. As cinco espécies de maior densidade, que totalizaram juntas $34,77 \%$, foram: Piptadenia gonoacantha (70 indivíduos/ha), Solanum leucodendron (52 indivíduos/ha), Apuleia leiocarpa (50 indivíduos/ha), Xylopia sericea (38 indivíduos/ha) e Sparattosperma leucanthum (32 indivíduos/ha).

Comparando as condições de floresta, observouse (Quadro 1) redução de 11,45\% no número de indivíduos por hectare na área da floresta explorada, porém tal diferença foi não-significativa $(P>0,05)$. Isso indica que a área da floresta explorada retomou o crescimento, de forma que o estoque explorado ficou sendo reposto.

Esses resultados concordam com o mencionado nos trabalhos de Souza (1999) e Tabarelli (1997). A exploração florestal, ao diminuir a densidade absoluta, ativa e acelera os processos de dinâmica sucessional. Resposta semelhante encontrou Tabarelli (1997), ao estudar diferentes trechos de floresta ombrófila densa, com 10, 18 e 40 anos de idade, e um trecho de floresta adulta, observando que nos trechos onde houve ação antrópica a estrutura horizontal evoluiu rapidamente em direção àquela encontrada na floresta madura.

Embora tenha sido estatisticamente igual $(\mathrm{P}>0,05)$, verificou-se (Quadro 2) que o número de indivíduos por hectare para o grupo de uso das espécies sem valor madeireiro (demais espécies) foi menor na área de floresta explorada. Segundo Hendrison (1990), a extensão dos danos à estrutura da floresta explorada é determinada pela intensidade de exploração. Ressalta-se que a exploração das árvores de grande tamanho forma 
Quadro 1 - Resumo da análise da variância das estimativas dos parâmetros densidade absoluta (DA), dominância absoluta (DoA) e volume por hectare de fuste comercial com casca (VoAfcc), nas áreas de florestas não-explorada e explorada, Fazenda Paciência, Matias Barbosa, MG

Table 1 - Summary of analysis of variance of the estimations of the parameters absolute density (DA), absolute dominance $(D o A)$ and volume per hectare of commercial log with bark (VoAfCC) for the unexploited and exploited forest areas, Fazenda Paciencia, municipality of Matias Barbosa, Minas Gerais State

\begin{tabular}{|c|c|c|c|c|}
\hline \multirow{2}{*}{ Fonte de Variação } & \multirow{2}{*}{ G L } & \multicolumn{3}{|c|}{ Quadrados Médios } \\
\hline & & $D A(\mathrm{~N} / \mathrm{ha})$ & $\operatorname{DoA}\left(\mathrm{m}^{2} / \mathrm{ha}\right)$ & $V o A_{f c c}\left(m^{3} / h a\right)$ \\
\hline Condições de floresta & 1 & $294,5335^{\text {ns }}$ & $2,558076^{*}$ & $114,0971^{*}$ \\
\hline Grupo uso & 2 & $19981,74^{*}$ & $15,69241^{*}$ & $469,8214^{*}$ \\
\hline Floresta x Grupo uso & 2 & $1200,134^{*}$ & $1,885767^{*}$ & $68,76851^{*}$ \\
\hline Resíduo & 24 & 257,4658 & 0,3614696 & 8,628947 \\
\hline CVexp. (\%) & & 32,658 & 48,067 & 45,222 \\
\hline
\end{tabular}

* = significativo a 5\% de probabilidade, pelo teste F; e ns = não-significativo a 5\% de probabilidade, pelo teste $\mathrm{F}$.

Quadro 2 - Médias de densidade das florestas não-explorada e explorada, Fazenda Paciência, Matias Barbosa, MG

Table 2 - Mean density for unexploited and exploited forests, Fazenda Paciência, municipality of Matias Barbosa, Minas Gerais State

\begin{tabular}{lrr}
\hline \multirow{2}{*}{ Grupo de Uso } & \multicolumn{2}{c}{ Médias da Densidade (No ind./ha) } \\
\cline { 2 - 3 } & Não-Explorada & Explorada \\
\hline Comerciais (C) & $114,000 \mathrm{Aa}$ & $87,200 \mathrm{Ab}$ \\
Potenciais (P) & $28,400 \mathrm{Ba}$ & $11,600 \mathrm{Ba}$ \\
Demais espécies (D) & $31,200 \mathrm{Ba}$ & $22,400 \mathrm{Ba}$ \\
\hline
\end{tabular}

Médias seguidas pela mesma letra maiúscula na coluna e minúscula na linha, respectivamente, não diferem entre si, pelo teste de Tukey a 5\% de probabilidade.

grandes clareiras, nas quais árvores de tamanho pequeno, arvoretas e mudas são destruídas. Por conseguinte, tal redução pode ser atribuída aos danos severos provocados pela exploração convencional, que no presente estudo foi de alta intensidade.

De acordo com os resultados, ficou, assim, evidente a necessidade de aplicação de tratamentos silviculturais, a fim de manter um estoque comercial na área de floresta explorada e, sobretudo, mitigar os impactos negativos da exploração florestal convencional e controlar a população de árvores e o crescimento da floresta.

\subsubsection{Dominância Absoluta (DoA $\left.A_{i}\right)$}

A floresta não-explorada apresentou uma área basal de $23,18 \mathrm{~m}^{2}$ por hectare, onde se observou que apenas cinco espécies representaram $49,88 \%$ de toda a área basal, sendo elas: Xylopia sericea (3,88 m²/ha), Piptadenia gonoacantha (2,21 m²/ha), Anadenanthera colubrina $\left(2,08 \mathrm{~m}^{2} / \mathrm{ha}\right)$, Ficus enormis $\left(1,82 \mathrm{~m}^{2} / \mathrm{ha}\right)$ e Platypodium elegans (1,58 m²/ha). Das espécies de maior área basal, Ficus enormis é representada por apenas dois indivíduos, figurando, entretanto, entre os maiores valores de área basal em função do tamanho significativo desses indivíduos.

Com relação à floresta explorada, observou-se um estoque de área basal de $14,44 \mathrm{~m}^{2}$ por hectare, onde as cinco espécies que representaram $43,04 \%$ de toda a área basal foram: Piptadenia gonoacantha $\left(2,03 \mathrm{~m}^{2} /\right.$ ha), Cecropia hololeuca $\left(1,24 \mathrm{~m}^{2} / \mathrm{ha}\right)$, Solanum leucodendron $\left(1,16 \mathrm{~m}^{2} / \mathrm{ha}\right)$, Xylopia sericea $\left(0,92 \mathrm{~m}^{2} / \mathrm{ha}\right)$ e Plathymenia foliolosa $\left(0,86 \mathrm{~m}^{2} / \mathrm{ha}\right)$.

Avaliando o estoque de área basal das duas condições de floresta, observou-se que, após seis anos decorridos da exploração florestal convencional, a área de floresta explorada (Quadro 1) ainda apresentava redução significativa $(\mathrm{P}<0,05)$ de área basal total. Além disso, o teste de Tukey (Quadro 3) apontou diferenças significativas $(\mathrm{P}<0,05)$ entre as médias de áreas basais das espécies madeireiras comerciais nas áreas de florestas não-explorada e explorada, respectivamente.

R. Árvore, Viçosa-MG, v.28, n.3, p. 407-417, 2004 
Quadro 3 - Médias de dominância das florestas não-explorada e explorada, Fazenda Paciência, Matias Barbosa, MG

Table 3 - Mean dominance for unexploited and exploited forests, Fazenda Paciência, municipality of Matias Barbosa, Minas Gerais State

\begin{tabular}{lcc}
\hline \multirow{2}{*}{ Grupo de Uso } & \multicolumn{3}{c}{ Médias da Dominância (m²/ha) } \\
\cline { 2 - 3 } & Não Explorada & Explorada \\
\hline Comerciais (C) & $3,49 \mathrm{Aa}$ & $1,91 \mathrm{Ab}$ \\
Potenciais (P) & $0,56 \mathrm{Ba}$ & $0,56 \mathrm{Ba}$ \\
Demais Espécies (D) & $0,58 \mathrm{Ba}$ & $0,40 \mathrm{Ba}$ \\
\hline
\end{tabular}

Médias seguidas pela mesma letra maiúscula na coluna e minúscula na linha, respectivamente, não diferem entre si, pelo teste de Tukey a 5\% de probabilidade.

Os dados permitem, portanto, inferir que a exploração madeireira convencional alterou significativamente ( $P$ $<0,05)$ o estoque remanescente de área basal na floresta explorada, principalmente das espécies madeireiras de valor comercial na região. Resultados semelhantes foram obtidos por Pinto (2000) e Pinto et al. (2002), também comparando áreas de florestas não-explorada e explorada via PMFS.

\subsubsection{Volume de Fuste com Casca (VoAfcc)}

A floresta não-explorada apresentou um volume de fuste total de $126,95 \mathrm{~m}^{3}$ por hectare, onde cinco espécies representaram $44,71 \%$ de todo o volume, sendo elas: Xylopia sericea $\left(22,69 \mathrm{~m}^{3} / \mathrm{ha}\right)$, Piptadenia gonoacantha (12,06 $\left.\mathrm{m}^{2} / \mathrm{ha}\right)$, Anadenanthera colubrina (11,44 m³a), Ficus enormis (10,58 m²/ha) e Plathymenia foliolosa $\left(7,62 \mathrm{~m}^{3} / \mathrm{ha}\right)$.

Com relação à floresta explorada, o estoque total de volume de fuste com casca foi de $68,37 \mathrm{~m}^{3}$ por hectare, onde as cinco espécies mais representativas, que perfizeram juntas 46,12\% do volume total da floresta explorada, foram: Piptadenia gonoacantha com 10,90 $\mathrm{m}^{3} / \mathrm{ha}$, Cecropia hololeuca $\left(6,54 \mathrm{~m}^{3} / \mathrm{ha}\right)$, Xylopia sericea (4,93 m³/ha), Solanum leucodendron $\left(4,83 \mathrm{~m}^{3} /\right.$ ha) e Apuleia leiocarpa (4,34 m³/ha).

Os resultados, portanto, permitem concluir que, após seis anos decorridos da atividade de exploração florestal convencional, houve uma redução de 46,14\% do volume total em relação à floresta não-explorada. Assim, a exploração convencional diminuiu significativamente $(\mathrm{P}<0,05)$ o estoque de volume na flo- resta explorada (Quadro 1), estando em consonância com os dados obtidos em uma floresta da Amazônia Ocidental (PINTO, 2000; PINTO et al. 2002). Essa mudança foi verificada, principalmente, nas espécies de valor comercial (Quadro 4).

Embora os resultados das estimativas dos parâmetros da estrutura por si sós não dêem total suporte, sugerem-se planejar e executar as operações de exploração mediante o uso de técnicas de exploração de impacto reduzido (EIR), avaliação da viabilidade de aplicação de tratamentos silviculturais e execução do monitoramento da floresta, a fim de verificar se a área de floresta explorada será capaz de restaurar o estoque original de área basal e o volume, conforme previsto no ciclo de corte de 12 anos, de acordo com o plano de manejo elaborado para a propriedade.

\subsection{Distribuição Diamétrica}

Observou-se que tanto a área de floresta nãoexplorada quanto a de floresta explorada apresentaram estrutura diamétrica com tendência típica de estrutura de florestal ineqüiânea, ou seja, em "J invertido" (MEYER, 1952).

Comparando a estrutura dos diâmetros de ambas as áreas de floresta, observou-se, no centro de classe de $12,5 \mathrm{~cm}$, uma concentração do número de árvores e de área basal nas áreas de florestas não-explorada e explorada (Figuras 2 e 3).

O resultado do teste F Graybill (GRAYBILL, 1976) evidenciou diferença não-significativa $(P>0,05)$ entre as distribuições de densidades por classe de diâme-

Quadro 4 - Médias de volume das florestas não-explorada e explorada, Fazenda Paciência, Matias Barbosa, MG

Table 4 - Mean volume for unexploited and exploited forests, Fazenda Paciência, municipality of Matias Barbosa, Minas Gerais State

\begin{tabular}{lcc}
\hline \multirow{2}{*}{ Grupo de Uso } & \multicolumn{2}{c}{ Médias de Volume (m³/ha) } \\
\cline { 2 - 3 } & Não-explorada & Explorada \\
\hline Comerciais (C) & $19,38 \mathrm{Aa}$ & $9,43 \mathrm{Ab}$ \\
Potenciais (P) & $3,05 \mathrm{Ba}$ & $2,37 \mathrm{Ba}$ \\
Demais espécies (D) & $2,90 \mathrm{Ba}$ & $1,83 \mathrm{Ba}$ \\
\hline
\end{tabular}

Médias seguidas de pelo menos uma mesma letra maiúscula na coluna e minúscula na linha, respectivamente, não diferem entre si, pelo de teste de Tukey a 5\% de probabilidade. 


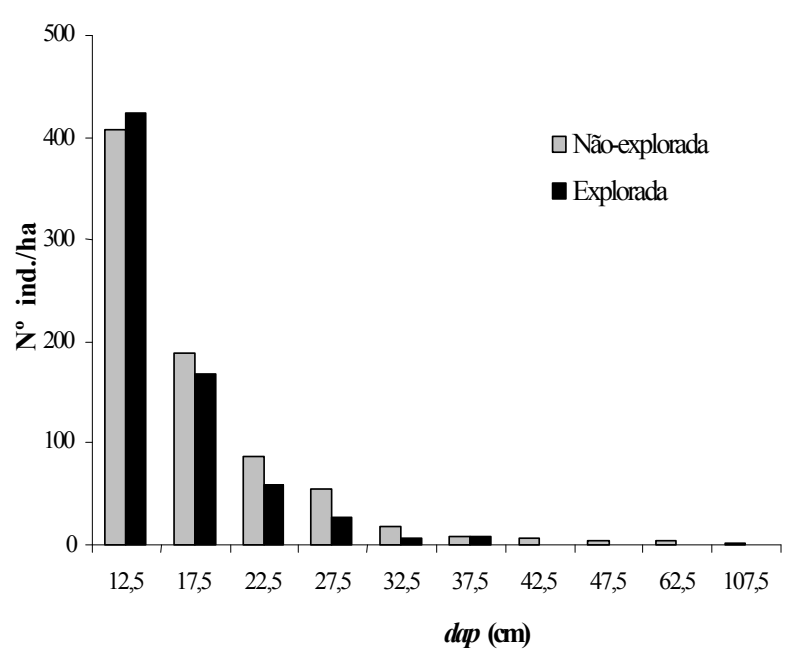

Figura 2 - Distribuição do número de indivíduos por hectare, por classe diamétrica, nas áreas de florestas nãoexplorada e explorada, Fazenda Paciência, Matias Barbosa, MG.

Figure 2 -Distribution of number of individuals per hectare per diameter classes in unexploited and exploited forests, Fazenda Paciência, municipality of Matias Barbosa, Minas Gerais State.

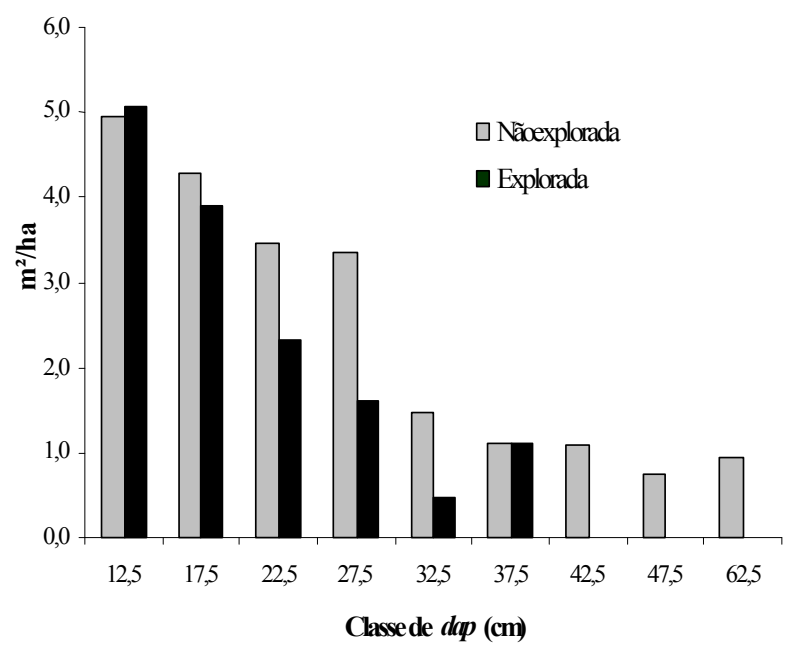

Figura 3 - Distribuição da área basal por hectare, por classe diamétrica, nas áreas de florestas não-explorada e explorada, Fazenda Paciência, Matias Barbosa, MG.

Figure 3 - Basal area distribution per hectare per diameter classes in unexploited and exploited forests, Fazenda Paciência , municipality of Matias Barbosa, Minas Gerais State. tro, entre as áreas de florestas não-explorada e explorada (Quadro 5). Porém, verificaram-se na floresta explorada as maiores reduções de densidade absoluta nas classes de maiores diâmetros, com a ressalva de que, a partir do centro de classe de $37,5 \mathrm{~cm}$, as reduções foram de $100 \%$ (Figura 2). As espécies com o maior potencial madeireiro na região sofreram queda em suas densidades, principalmente nas classes de maior diâmetro, destacando-se: Anadenanthera colubrina, Apuleia leiocarpa, Machaerium nyctitans e Platypodium elegans que apresentaram, nas condições de exploração convencional, indivíduos apenas até a classe de 22,5 $\mathrm{cm}$.

As espécies de maior valor comercial madeireiro como Anadenanthera colubrina, Plathymenia foliolosa, Platypodium elegans, Xylopia sericea e Machaerium nyctitans sofreram diminuições de área basal, em quase todas as classes diamétricas, superiores a $60 \%$. Apuleia leiocarpa e Piptadenia gonoacantha, por sua vez, exibiram reduções superiores a $60 \%$ apenas nas classes de maiores diâmetros.

Após seis anos decorridos das atividades de exploração florestal convencional, observou-se que, a partir do centro de classe de $37,5 \mathrm{~cm}$, as reduções de área basal na área de floresta explorada foram de $100 \%$ (Figura 3 ), em comparação com à estrutura da área de floresta não-explorada, o que ultrapassa os níveis de intervenção permitidos pela Portaria 054, do Instituto Estadual de Florestas (IEF-MG, 1997), que regulamenta que essas intervenções não devem exceder a $60 \%$ da área basal das tipologias florestais, por classe diamétrica e por espécie.

Quadro 5-Resultado do teste F Graybill , a 5\% de probabilidade, das distribuições diamétricas, nas áreas de florestas não-explorada e explorada, Fazenda Paciência, Matias Barbosa, MG

Table 5 - F Graybill test result, at $5 \%$ probability level, of diametric distributions, for the unexploited and exploited forests, Fazenda Paciência, municipality of Matias Barbosa, Minas Gerais State

\begin{tabular}{lcc}
\hline Verificadores & Centro de Classes* & $\mathrm{F}(\mathrm{Ho})$ \\
\hline $\begin{array}{l}\text { Densidade } \\
\text { absoluta (N/ha) }\end{array}$ & 12,5 até 107,5 & $2,76812 \mathrm{~ns}$ \\
\hline $\begin{array}{l}\text { Dominância } \\
\text { absoluta (m2/ha) }\end{array}$ & 12,5 até 107,5 & $8,890411 *$ \\
\hline
\end{tabular}

* Classe com amplitude de $5 \mathrm{~cm}$. 


\subsection{Estrutura Interna}

\subsubsection{Infestação de Cipós}

A avaliação quanto à infestação de cipós (Quadro 6) apontou alta incidência, tanto na área de floresta não-explorada quanto na área de floresta explorada. De acordo com Budowski (1966), os cipós são mais abundantes nos primeiros estágios sucessionais, diminuindo a sua densidade à medida que a floresta tende ao estádio climático. Almeida Junior (1999), por sua vez, estudando o estado de conservação de fragmentos secundários de Floresta Estacional Semidecidual em Viçosa, MG, encontrou valores similares de infestação de cipós com 72,73\% dos indivíduos amostrados com alguma infestação de cipós.

De acordo com Almeida (1996), os níveis de infestação de cipós são importantes parâmetros no diagnóstico do estado de conservação de um fragmento florestal. Ele acrescentou que a abertura de clareiras e a exploração seletiva de madeira, assim como outras interferências antrópicas, podem aumentar a incidência de cipós num fragmento florestal.

Os dados obtidos indicaram, como recomendação, a adoção de um manejo de baixo impacto que vise, no mínimo, a uma exploração mais conservativa, em razão de se tratar de uma área de Floresta Estacional Semidecidual Secundária, ou seja, já afetada historicamente por fatores antrópicos. Além disso, ressal-

Quadro 6 - Distribuição do número de indivíduos e percentagens por infestação de cipós, nas áreas de florestas não-explorada e explorada, Fazenda Paciência, Matias Barbosa, MG

Table 6 - Distribution of number of individuals and percentage of infestation by vines in unexploited and exploited forests, Fazenda Paciência, municipality of Matias Barbosa, Minas Gerais State

\begin{tabular}{lrrrr}
\hline \multirow{2}{*}{ Infestação de Cipós Não-explorada } & \multicolumn{2}{c}{ Explorada } \\
\cline { 2 - 5 } \multicolumn{2}{c}{$\mathrm{N}^{\circ}$ ind./ha } & $\%$ & $\mathrm{~N}^{\circ}$ ind./ha & $\%$ \\
\hline Nenhuma infestação & 200 & 25,4 & 150 & 21,6 \\
Apenas no tronco & 90 & 11,5 & 74 & 10,6 \\
Apenas na copa & 100 & 12,7 & 64 & 9,2 \\
No tronco e na copa & 396 & 50,4 & 408 & 58,6 \\
Total & 786 & 100,0 & 696 & 100,0 \\
\hline
\end{tabular}

tam-se a necessidade e obrigatoriedade de planejamento das operações de exploração de baixo impacto, bem como prescrição e aplicação de tratamentos silviculturais, principalmente corte pré-exploratório de cipós, a fim de diminuir os riscos durante as atividades de exploração e os impactos negativos na vegetação remanescente.

\subsubsection{Posição de Danos}

Na floresta explorada foram observados $40,2 \%$ dos indivíduos com danos no tronco e na parte comercializável da madeira, implicando um comprometimento do valor comercial do estoque remanescente da floresta explorada (Quadro 7) e indicando não estar havendo planejamento das operações de exploração de madeira.

Os danos ao tronco encontrados na floresta explorada podem ser considerados alarmantes quando comparados com os observados por Pinto (2000) e Pinto et al. (2002), que, avaliando os danos causados à vegetação adulta numa área explorada na Amazônia Ocidental, encontraram apenas 8\% de árvores danificadas, entre tronco e copa. Os referidos autores mencionaram que essas são percentagens realmente baixas e que tal resultado ocorreu em função, principalmente, do planejamento das operações de préexploração, corte de cipós, abertura das trilhas de arraste e direcionamento de queda, além, é claro, de se tratar de uma fitofisionomia e posições topográficas distintas.

Quadro 7 - Distribuição do número de indivíduos e percentagens, por posição de danos, das áreas de florestas nãoexplorada e explorada, Fazenda Paciência, município de Matias Barbosa, MG

Table 7 - Distribution of number of individuals and percentage of damage, per position, in unexploited and exploited forests, Fazenda Paciência, municipality of Matias Barbosa, Minas Gerais State

\begin{tabular}{|c|c|c|c|c|}
\hline \multirow{2}{*}{ Posição de Danos } & \multicolumn{2}{|c|}{ Não-explorada } & \multicolumn{2}{|c|}{ Explorada } \\
\hline & $\mathrm{N}^{\mathrm{o}}$ ind./ha & $\%$ & $\mathrm{~N}^{\mathrm{o}}$ ind./ha & $\%$ \\
\hline Nenhum dano & 426 & 54,2 & 234 & $\overline{33,6}$ \\
\hline Danos no tronco & 172 & 21,9 & 280 & 40,2 \\
\hline Danos na copa & 188 & 23,9 & 182 & 26,2 \\
\hline Total & 786 & 100,00 & 696 & 100,00 \\
\hline
\end{tabular}




\subsubsection{Causa de Danos no Fuste}

Na floresta explorada, verificou-se que $65,8 \%$ dos indivíduos remanescentes apresentavam danos devidos às atividades de exploração florestal convencional, número considerado alto quando comparado com os dados obtidos por Martins (1995) e Martins et al. (2003), que, avaliando os danos à vegetação adulta após as atividades de exploração, constataram que 80\% dos indivíduos não apresentavam danos.

Já na área de floresta não-explorada, 42,0\% dos indivíduos apresentaram danos devidos às atividades de exploração florestal, conforme observado em campo (Quadro 8). Assim, os resultados evidenciaram o fato de que não foram respeitados os limites dos talhões previstos para o corte anual, ocorrendo exploração de madeira também na área da floresta nãoexplorada. Além disso, os resultados indicam a ausência de planejamento das atividades de exploração florestal, redundando em um número alto de árvores danificadas e comprometendo a saúde e a qualidade do estoque remanescente.

\subsubsection{Infestação de Bambus}

$\mathrm{Na}$ área de floresta não-explorada, apenas 0,5\% dos indivíduos apresentaram infestação de bambus, ao passo que na área de floresta explorada $26,5 \%$ dos indivíduos sofreram algum tipo de infestação de bambus em função provavelmente, da abertura de grandes clareiras durante as atividades de exploração florestal, favorecendo a infestação de bambus nativos em algumas áreas, conforme observado in loco (Quadro 9). Com isso, foi necessário o controle da população

Quadro 8 - Distribuição do número de indivíduos e percentagens, por qualidade de danos, das áreas de florestas nãoexplorada e explorada, Fazenda Paciência, Matias Barbosa, MG

Table 8 - Distribution of number of individuals and percentage per quality of damage in unexploited and exploited forests, Fazenda Paciência , municipality of Matias Barbosa, Minas Gerais State

\begin{tabular}{lcrrr}
\hline \multirow{2}{*}{ Qualidade de danos } & \multicolumn{2}{c}{ Não-explorada } & \multicolumn{2}{c}{ Explorada } \\
\cline { 2 - 5 } & $N^{\circ}$ ind./ha & \multicolumn{1}{c}{$\%$} & $\mathrm{~N}^{\circ}$ ind./ha & $\%$ \\
\hline Nenhum dano & 426 & 54,2 & 234 & 33,6 \\
Causas naturais & 30 & 3,8 & 4 & 0,6 \\
Devido à exploração & 330 & 42,0 & 458 & 65,8 \\
Total & 786 & 100,0 & 696 & 100,0 \\
\hline
\end{tabular}

dessa espécie de bambu nas áreas infestadas, a fim de diminuir a competição e os danos à vegetação remanescente.

Quadro 9 - Distribuição do número de indivíduos e percentagens, por infestação de bambus, das áreas de florestas não-explorada e explorada, Fazenda Paciência, município de Matias Barbosa, MG

Table 9 - Distribution of number of individuals and percentage of bamboo infestation in unexploited and exploited forests, Fazenda Paciência, municipality of Matias Barbosa, Minas Gerais State

\begin{tabular}{|c|c|c|c|c|}
\hline \multirow{2}{*}{ nfestação de Bambus } & \multicolumn{2}{|c|}{ Não-explorada } & \multicolumn{2}{|c|}{ Explorada } \\
\hline & $\mathrm{N}^{\mathrm{o}}$ ind./ha & $\%$ & $\mathrm{~N}^{\mathrm{o}}$ ind./ha & $\%$ \\
\hline Nenhuma infestação & 782 & 99,5 & 512 & 73,5 \\
\hline Apenas no tronco & 4 & 0,5 & 36 & 5,2 \\
\hline No tronco e na copa & 0 & 0,0 & 148 & 21,3 \\
\hline Total & 786 & 100,0 & 696 & 100,0 \\
\hline
\end{tabular}

\section{CONCLUSÕES}

A exploração madeireira convencional alterou significativamente $(\mathrm{P}<0,05)$ os estoques totais de área basal e de volume da área de floresta explorada, ressaltando-se que os maiores impactos ocorreram na estrutura das espécies de maior valor comercial madeireiro.

As espécies de maior valor comercial madeireiro foram Anadenanthera colubrina, Plathymenia foliolosa, Platypodium elegans, Xylopia sericea e Machaerium nyctitans. Elas sofreram diminuições nos estoques de área basal e de volume na floresta explorada convencionalmente, em quase todas as classes diamétricas, superiores a $60 \%$, o que viola as condições impostas pela legislação florestal de Minas Gerais.

\section{REFERÊNCIAS BIBLIOGRÁFICAS}

\section{ALMEIDA JUNIOR, J.S. Florística e fitossociologia de um fragmento de floresta estacional semidecidual, Viçosa, Minas Gerais. 1999. 148f. Dissertação (Mestrado em Ciência Florestal) - Universidade Federal de Viçosa, Viçosa, 1999.}


ALMEIDA, D.S. Florística e estrutura de um fragmento de floresta atlântica, no município de Juiz de Fora. 1996. 91f. Dissertação (Mestrado em Ciência Florestal) Universidade Federal de Viçosa, Viçosa, 1996.

BUDOWSKI, G. Los bosques de los trópicos húmedos de América. Turrialba v.16, n. 1, p. 42-52, 1966.

COELHO, D.J. Modelo de gestão florestal sustentável para a microrregião de Viçosa, Minas Gerais. 1999. $80 \mathrm{f}$. Dissertação (Mestrado em Ciência Florestal) Universidade Federal de Viçosa, Viçosa, 1999.

Embrapa Brasileira de Pesquisa Agropecuária EMBRAPA. Diagnóstico dos projetos de manejo florestal no Estado do Pará Fase Paragominas. Belém: CPATU, 1996. 99 p.

GRAYBILL, F. A. Theory and application of the linear model. Massacusetts: Belmont Duxbury Press, 1976. 704 p.

HENDRISON, U. J. Damage-controlled logging in managed tropical rain forest in Suriname. Wageningen: Agricultural University, 1990. 204 p.

HERZOG, W. Economia florestal na Fazenda Paciência (Matias Barbosa, MG). Ministério da Agricultura. Arquivos do Serviço

Florestal, v.11, p 22, 1957.

HOSOKAWA, R.T.; MOURA, J.B.; CUNHA, U.S. Introdução ao manejo e economia de florestas. Curitiba: UFPR, 1998.162 p.

IBAMA. Processo de avaliação de planos de manejo florestal sustentável. Disponível em: <http://www.ibama.gov.br/atuação/ flores/deref/manflor/metodo.html>. Acesso em: 2000.

R. Árvore, Viçosa-MG, v.28, n.3, p. 407-417, 2004
LILLIEFORS, H. W. On the kolmogorov-smirnov test for normality with mean and variance unknown. Jornal of the American Statistical Association, v. 64, p. 399-402, 1967.

MARTINS, S.S. Efeitos da exploração madeireira no solo, na florística e estrutura de uma floresta estacional semidecidual no sudoeste do Paraná. 1995. 119f. Tese (Doutorado em Ciência Florestal)Universidade Federal de Viçosa, Viçosa, 1995.

MARTINS, S. S. et al. Efeito da exploração florestal seletiva em uma floresta estacional semidecidual. Revista Árvore, v.27, n.1, p. 6570, 2003.

MEYER, H.A. Structure, growth, and drain in balanced uneven-aged forests. Journal Forest, v. 50, n. 2, p. 85-92, 1952.

MUELLER-DOMBOIS, D.; ELLENBERG, H. Aims and methods of vegetation ecology. New York: John Wiley e Sons, 1974. 547 p.

PINTO, A.C. Análise dos danos da exploração de madeira em floresta tropical úmida sob regime de manejo florestal sustentável, na Amazônia Ocidental. 2000. 131f. Dissertação (Mestrado em Ciência Florestal) - Universidade Federal de Viçosa, Viçosa, 2000.

PINTO, A. C. M. et al. Análise de danos de colheita de madeira em floresta tropical úmida sob regime de manejo florestal sustentado na Amazônia Ocidental. Revista Árvore, v.26, n.4, p. 459-466, 2002.

RODRIGUEZ, L.C.E. Monitoramento florestal: iniciativas, definições e recomendações. IPEF, v.12, n. 31, p. 9-21, 1998. (Série técnica).

SILVA, J.N.M.; LOPES, J. C. A. Inventário

florestal contínuo em florestas tropicais: a metodologia utilizada pela EMBRAPA-CPATU na Amazônia brasileira. Belém: EMBRAPA, 1984. 39 p. 
SOUZA, A.L. Estrutura, dinâmica e manejo de florestas. Viçosa, MG: UFV, 2004. $40 \mathrm{p}$

SOUZA, A.L. et al. Avaliação dos impactos das operações de exploração de madeira e de tratamentos silviculturais num projeto de manejo sustentável. Viçosa, MG: 1998. 248 p.(Relatório Técnico de Pesquisa - CNPq, Processo 520010/965 - Universidade Federal de Viçosa, Viçosa.
TABARELLI, M. A regeneração da floresta atlântica montana. 1997. 104f. Tese (Doutorado em Engenharia Florestal) Universidade de São Paulo, São Paulo, 1997.

VELOSO, H.P. Manual técnico da vegetação brasileira. Rio de Janeiro: IBGE, 1992. 92 p. (Série Manuais Técnicos de Geociências, 1).

WINER, B. J. Statistical principles in experimental design. New York: McgrawHill, 1962, p. 94. 